\title{
Detection of DNA damage response in nonalcoholic fatty liver disease via p53-binding protein 1 nuclear expression
}

\author{
Yuko Akazawa ${ }^{1,2} \cdot$ Ryoma Nakashima $^{1} \cdot$ Katsuya Matsuda $^{3} \cdot$ Koji Okamaoto $^{1} \cdot$ Ran Hirano $^{1} \cdot$ Hiroko Kawasaki $^{1} \cdot$

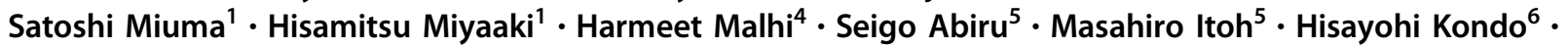 \\ Junya Fukuoka ${ }^{2} \cdot K_{a z u h i k o}$ Nakao $^{1} \cdot$ Masahiro Nakashima $^{3}$
}

Received: 3 July 2018 / Revised: 14 January 2019 / Accepted: 15 January 2019 / Published online: 26 February 2019

(c) United States \& Canadian Academy of Pathology 2019

\begin{abstract}
Nonalcoholic fatty liver disease is a major liver disease that leads to cirrhosis and/or hepatocellular carcinoma in a subset of patients. The mechanism underlying disease progression is largely unknown. p53-binding protein 1 (53BP1) is a DNA damage response protein that rapidly localizes at the site of DNA double-strand breaks. In this study, we investigated nuclear 53BP1positive foci formation as an indicator of DNA double-strand breaks in human nonalcoholic fatty liver disease liver tissues by immunofluorescence microscopy. A total of 52 liver tissue samples, including 43 nonalcoholic fatty liver disease samples and 9 controls, were studied. Our results show that the number of abnormal 53BP1-positive foci in hepatocytes (defined as three or more discrete nuclear foci and/or large foci greater than $1 \mu \mathrm{M}$ ) was significantly increased in nonalcoholic fatty liver disease patients compared to that in controls, both in nonalcoholic fatty liver $(p<0.01)$ and nonalcoholic steatohepatitis patients $(p<$ 0.01). The number of large foci was significantly increased in the nonalcoholic steatohepatitis cases compared to that in the nonalcoholic fatty liver cases $(p<0.05)$ and correlated with increased stage of fibrosis. The number of large-foci-expressing hepatocytes was positively correlated with increased age $(p<0.01)$ and negatively correlated with serum platelet count $(p<$ 0.05). In addition, we performed an in vitro assay using rat hepatocytes treated with the saturated free fatty acid palmitate. Treatment appeared to augment the number of abnormal foci, indicating an induction of double-strand breaks in the hepatocytes through free fatty acid treatment in a caspase-dependent manner. This study demonstrates that 53BP1-positive nuclear foci formation is associated with disease progression in nonalcoholic fatty liver disease patients. Analysis of 53BP1 expression might be a feasible technique to estimate genomic instability in nonalcoholic fatty liver disease.
\end{abstract}

Supplementary information The online version of this article (https:// doi.org/10.1038/s41379-019-0218-8) contains supplementary material, which is available to authorized users.

Yuko Akazawa

akazaway@nagasaki-u.ac.jp

1 Department of Gastroenterology and Hepatology, Nagasaki University Graduate School of Biomedical Sciences,

Nagasaki, Japan

2 Department of Pathology, Nagasaki University Graduate School of Biomedical Sciences, Nagasaki, Japan

3 Department of Tumor and Diagnostic Pathology, Atomic Bomb Disease Institute, Nagasaki University, Nagasaki, Japan

4 Division of Gastroenterology and Hepatology, Mayo Clinic, Rochester, MN, USA

5 Clinical Research Center, National Hospital Organization, Nagasaki Medical Center, Omura, Japan

6 Biostatistics Section, Division of Scientific Data Registry, Atomic Bomb Disease Institute, Nagasaki University, Nagasaki, Japan

\section{Introduction}

Nonalcoholic fatty liver disease is a major health problem because of its increasing prevalence and associated risk of progression to liver cirrhosis and hepatocellular carcinoma $[1,2]$. Although presence of fibrosis is associated with cancer development in nonalcoholic fatty liver disease patients [3, 4], hepatocellular carcinoma may also arise in noncirrhotic nonalcoholic fatty liver disease patients with mild or even no fibrosis [5]. The molecular mechanisms underlying nonalcoholic fatty liver disease progression and pathogenesis, including carcinogenesis, are largely unknown. Genomic instability is induced by functional abnormalities in the DNA damage response (DDR) pathway and plays a significant role in human carcinogenesis [6-8]. For example, exposure to genotoxic agents, such as ionizing radiation or ethanol metabolites, induces DNA doublestrand breaks $[9,10]$ and activates the DDR pathway to 
maintain genomic integrity. However, in the case of severe double-strand breaks, the DDR pathway is insufficient to properly repair the damaged DNA, which results in genomic instability. Such detrimental changes to genome stability are considered to play a central role in the carcinogenic process. Thus, extensive activation of the DDR pathway is often regarded as a hallmark of carcinogenesis.

p53-binding protein 1 (53BP1) belongs to the evolutionarily conserved DDR family of proteins that carry the breast cancer type 1 (BRCA1) C-terminal domains [11]. Since 53BP1 localizes at double-strand break sites and rapidly forms nuclear foci to activate downstream effectors, such as p53 tumor suppressor molecules, the presence of 53BP1 nuclear foci can be used as a reliable indicator of double-strand break sites and of DDR activation [12-14]. We previously demonstrated that in formalin-fixed, paraffin-embedded, immunofluorescently stained mouse intestine samples, 53BP1 nuclear foci formation and DDR are induced by radiation [15]. Since one manifestation of genomic instability is the induction of endogenous DDR, 53BP1 nuclear foci formation can be considered a histopathologic marker for genomic instability.

An association between DDR protein expression, including that of 53BP1 and $\gamma$-histone-2AX $(\gamma \mathrm{H} 2 \mathrm{AX})$, and the progression of genomic instability was recently reported in an animal model of inflammation-associated cancer [16]. Furthermore, our previous studies show that the number of 53BP1 nuclear foci increases in precancerous lesions, such as those observed in uterine cervical intraepithelial neoplasia [17], actinic keratosis of the skin [16], and thyroid follicular adenoma [15, 18]. It appears that the presence of three or more discrete nuclear foci and/or foci more than $1.0 \mu \mathrm{m}$ in diameter (large foci type) are closely associated with a higher level of cervical carcinogenesis [17], suggesting that the 53BP1 expression pattern is indeed beneficial in estimating the malignant potential and genomic instability of neoplasia. However, the expression status of DDR proteins in human nonalcoholic fatty liver disease hepatocytes is poorly understood.

Free fatty acids, especially saturated free fatty acids, such as palmitate, play a crucial role in nonalcoholic fatty liver disease pathogenesis [19-24]. Free fatty acids disrupt membrane stability in hepatocytes, leading to endoplasmic stress, mitochondrial dysfunction, and apoptosis $[25,26]$. However, the effect of saturated free fatty acids on double-strand breaks and DDR protein expression has not been evaluated. In this study, we analyzed DDR expression in human nonalcoholic steatohepatitis hepatocytes and in rat primary hepatocytes challenged with free fatty acids. The results of this study demonstrate the existence of a 53BP1 DDR in human nonalcoholic fatty liver disease in association with clinicopathological features. In addition, our findings highlight the role of free fatty acids in the induction of double-strand breaks and 53BP1 expression in vitro.

\section{Material and methods}

\section{Patients}

Patients who underwent liver biopsy between June 2009 and May 2015 and were both clinically and pathologically diagnosed with nonalcoholic fatty liver disease at the Nagasaki University Hospital were enrolled in this study. The biopsies were performed for other medical reasons and not for research purposes only. The study was conducted according to the Declaration of Helsinki and was approved by the Ethical Committee of the Nagasaki University Hospital (Office of Human Subjects Protection, Registration number: 1642509). Following the guidelines of the ethical committee's official informed consent and disclosure system, detailed information regarding the study was made available on the institution's website. Patients were able to withdraw their consent to participate in the study by following the instructions listed on the faculty website. A total of 60 patients were enrolled in the study. The following criteria were used to exclude patients from the study: under 20 years of age; significant alcohol consumption (greater than $20 \mathrm{~g}$ /day of ethanol); possibility of drug-induced liver dysfunction; type $\mathrm{A}, \mathrm{B}$, or $\mathrm{C}$ hepatitis infection; and/or steroid use.

Following these criteria, 43 nonalcoholic fatty liver disease patients (age 51.8 $\pm 14.3,16$ male and 27 female) were considered eligible for further analysis. As a normal control, nine liver biopsies and surgical cases (average age $68.0 \pm$ 11.3, 4 male and 5 female) were also analyzed from patients with histologically normal livers, meaning there was no detection of steatosis, inflammation, or fibrosis. Clinical data, including serum aspartate aminotransferase, alanine aminotransferase, platelet count, ferritin, and C-reactive protein as well as body mass index, were also measured during routine clinical analysis.

\section{Histopathological examination of the liver}

Liver biopsy was percutaneously performed with a 16gauge needle under the guidance of ultrasonography. The specimens were fixed in $10 \%$ formalin and were embedded in paraffin for histopathological examination. Two pathologists confirmed the nonalcoholic fatty liver disease diagnosis (YA and HM) based on the presence of steatosis $(\geq 5 \%)$. Nonalcoholic fatty liver disease was classified into nonalcoholic fatty liver or nonalcoholic steatohepatitis according to the Matteoni classification, 
as follows [27]: type 1, steatosis alone; type 2, steatosis with lobular inflammation; type 3 , steatosis with hepatocyte ballooning; and type 4 , features of type 3 plus Mallory-Denk bodies and/or fibrosis. Types 1 and 2 were diagnosed as nonalcoholic fatty liver $(n=6)$, and types 3 and 4 were diagnosed as nonalcoholic steatohepatitis $(n=37)$. In addition, to estimate the relationship between the 53BP1-positive foci and specific pathological features of nonalcoholic fatty liver disease, the nonalcoholic fatty liver disease activity score [28], which can range from 0 to 8 based on the sum of steatosis grade (0-3), lobular inflammation (0-3), and hepatocyte ballooning (0-2) [28], were assessed. Fibrosis scoring was performed according to the Nonalcoholic Steatohepatitis Clinical Research Network System as follows: 0 (absent), 1 (perisinusoidal/pericellular fibrosis), 2 (periportal fibrosis), 3 (bridging fibrosis), and 4 (cirrhosis) [28]. The degrees of portal inflammation were additionally assessed as described by Brunt et al. [29] (0 none; 1 mild; 2 moderate; or 3 severe). All the histological data were determined in agreement with two independent hepatopathologists (HM and YA).

\section{Immunofluorescence analysis}

Following deparaffinization and antigen retrieval by microwave treatment in citrate buffer ( $\mathrm{pH} \mathrm{6.0),} \mathrm{the} \mathrm{tissue}$ sections were preincubated with $10 \%$ normal goat serum. Tissues were then either incubated with a mixture of polyclonal rabbit anti-53BP1 antibody (diluted 1:1000; Bethyl Laboratories, Montgomery, TX, USA) and antihepatocyte mouse monoclonal antibody (diluted 1:1000; DaKo M7158, Santa Clara, CA, USA) or with a monoclonal mouse anti- $\gamma \mathrm{H} 2 \mathrm{AX}$ antibody (diluted 1:1000; ab26350; Abcam, Tokyo, Japan). The samples were subsequently incubated with Alexa Fluor 488-conjugated goat anti-rabbit and/or Alexa Fluor 594F(ab')-conjugated goat anti-mouse antibodies (Molecular Probes Inc., Eugene, OR, USA) as appropriate and mounted using VECTASHIELD ${ }^{\circledR}$ HardSet Mounting Medium containing 4',6-diamidino-2-phenylindole, dihydrochloride (DAPI; VECTOR Labs, Burlingame, CA, USA). The stained sections were photographed at $\times 100$ magnification and analyzed with a High Standard All-in-One Fluorescence Microscope (Biorevo BZ-9000; Keyence Japan, Osaka, Japan). 53BP1 immunoreactivity was classified using the following definitions based on a previous study [17]: (1) normal type, one or two discrete nuclear foci less than 1.0 $\mu \mathrm{m}$; (2) high DDR type, three or more discrete nuclear foci less than $1.0 \mu \mathrm{m}$; or (3) large foci type, discrete nuclear foci greater than $1.0 \mu \mathrm{m}$ (Fig. 1). The percentage of hepatocyte-positive nuclei containing each type of staining was calculated.

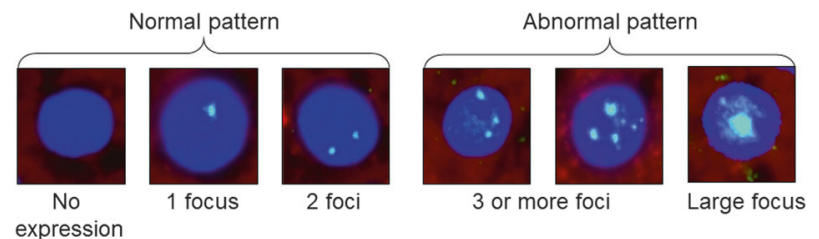

Fig. 1 Representative images of the different classifications of p53binding protein 1 (53BP1) expression in hepatocytes: normal/stable pattern (faint and diffuse nuclear staining); abnormal pattern (one or two discrete nuclear foci); and large foci (greater than $1 \mu \mathrm{m}$ in diameter)

\section{Rat hepatocyte cell culture}

Clone 9 cells, a cultured rat hepatocyte cell line, which was purchased from the American Type Culture Collection (Manassas, VA, USA), was employed in our in vitro study. Cells were maintained in F12-K medium (American Type Culture Collection) containing $10 \%$ fetal bovine serum.

\section{Free fatty acid treatment}

Palmitate was purchased from Sigma-Aldrich (St. Louis, MO, USA) and dissolved in isopropyl alcohol at a concentration of $20 \mathrm{mM}$. This solution was then added to Dulbecco's modified Eagle medium containing 1\% bovine serum albumin to maintain the physiological ratio between the bound and unbound palmitate in the medium. The palmitate concentration used in these experiments ranged from 0 to $100 \mu \mathrm{M}$. Cultured hepatocytes were treated with either palmitate or vehicle (isopropyl alcohol) for $24 \mathrm{~h}$, followed by fixation with $4 \%$ paraformaldehyde and preincubation with $10 \%$ normal goat serum. Cells were then treated according to the same immunofluorescence staining method described above.

\section{Quantification of apoptosis}

Apoptosis was quantified using DAPI (Sigma-Aldrich) in conjunction with a High Standard All-in-One Fluorescence Microscope (BZ-9000, Keyence Japan). Cells were stained with $5 \mu \mathrm{g} / \mathrm{mL}$ DAPI at $37^{\circ} \mathrm{C}$ for $30 \mathrm{~min}$. Apoptotic cells were morphologically assessed by fluorescence microscopy. Photographs were acquired from three random fields per sample, and apoptosis was quantified as a percentage of the total number of cells. At least 100 cells were counted in each field.

\section{BP1 expression in cultured hepatocytes}

Cells were washed with phosphate-buffered saline (PBS) and fixed with $4 \%$ paraformaldehyde in PBS for $15 \mathrm{~min}$. The cells were permeabilized using $0.0125 \%$ (w/v) 3-[(3- 
(a)

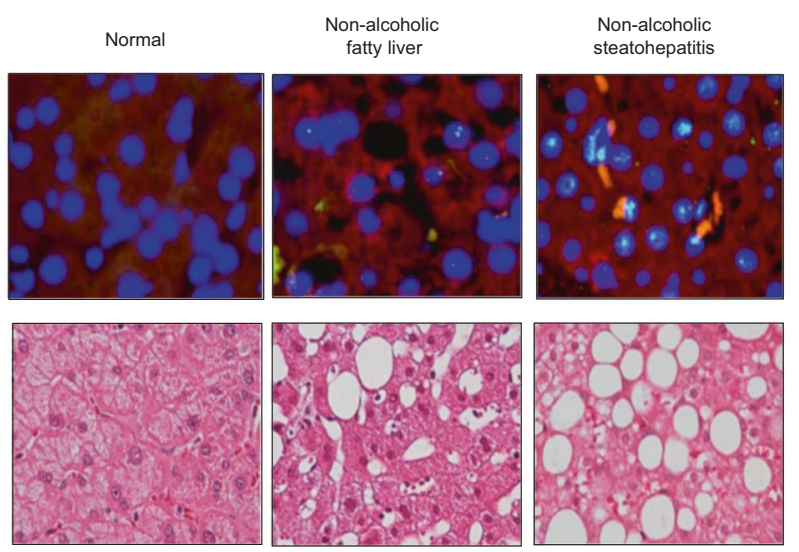

(c)

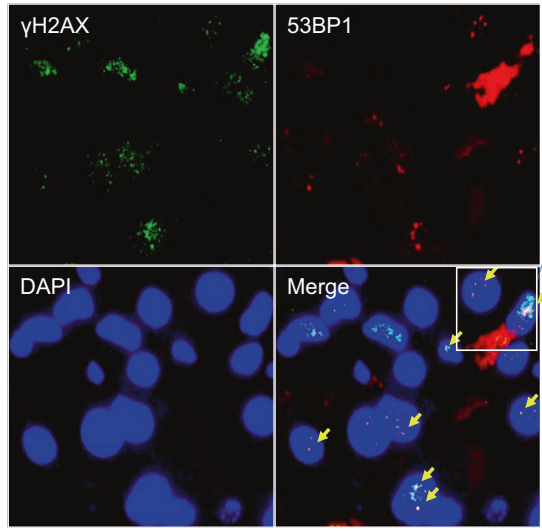

(e)

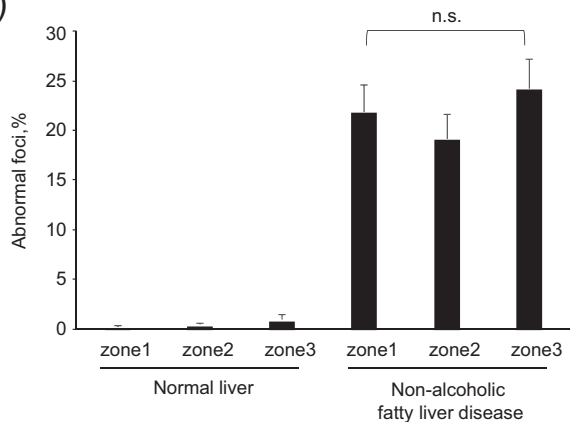

cholamidopropyl) dimethylammonio]-1-propanesulfonate (CHAPS) in PBS at $37^{\circ} \mathrm{C}$ for $10 \mathrm{~min}$. Cells were then washed with PBS and incubated in PBS containing 5\% goat
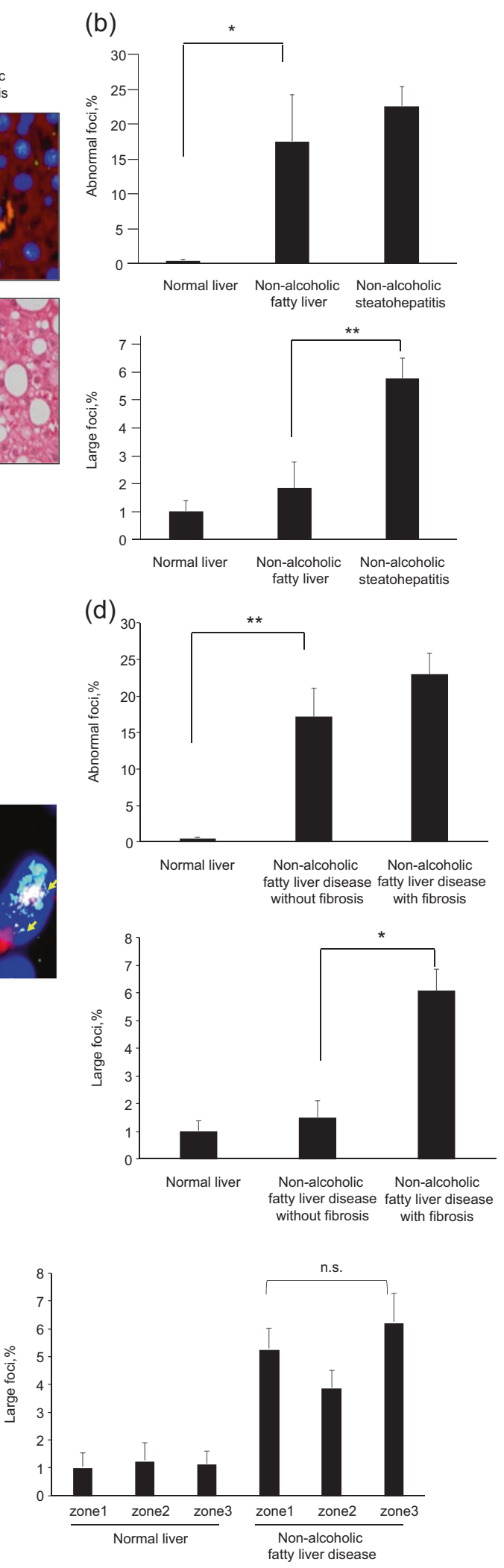

serum at room temperature for $1 \mathrm{~h}$. After incubation with anti-53BP1 antibody at $4{ }^{\circ} \mathrm{C}$ overnight, the cells were washed three times with PBS and then incubated with Alexa Fluor 
Fig. 2 p53-binding protein 1 (53BP1) expression in nonalcoholic fatty liver disease (nonalcoholic fatty liver disease) patients. a 53BP1 expression (green) in normal, nonalcoholic fatty liver, and nonalcoholic steatohepatitis liver tissues. The nuclei were visualized with 4",6-diamidino-2-phenylindole, dihydrochloride (blue), whereas an antihepatocyte antibody was used to indicate the cell cytoplasm (red). b Quantification of abnormal 53BP1-positive foci (upper panel) and large 53BP1-positive foci (lower panel). Data were calculated as a percentage $(\%)$ of the total number of hepatocytes per ( $n=9$ fields) and are presented as the means \pm SEM. $* p<0.01$, $* * p<0.05$. c Coimmunofluorescence of 53BP1 and gamma-histone-2AX $(\gamma \mathrm{H} 2 \mathrm{AX})$. Arrows indicate the coexpression sites. $\mathbf{d}$ The number of abnormal foci (upper) and large foci (lower panel) in normal and nonalcoholic fatty liver disease livers with or without the presence of fibrosis. Presence or absence of fibrosis was determined by Azan staining of the specimen. Data are expressed as the means \pm SEM. $* p<0.01, * * p<0.05$. e The number of abnormal foci (lower left panel) and large foci (lower right panel) in normal and nonalcoholic fatty liver disease livers among the three hepatic zones. Data are expressed as the means \pm SEM

488-conjugated goat anti-rabbit IgG (Molecular Probes) at $37^{\circ} \mathrm{C}$ for $2 \mathrm{~h}$. All of the antibodies were diluted in PBS containing 5\% fetal bovine serum. The cells were then washed and mounted onto slides using a ProLong ${ }^{\mathrm{TM}}$ Antifade kit with DAPI (Molecular Probes). The cells were imaged and analyzed with a fluorescence microscope.

\section{Caspase-3/7 activity assay}

Activation of caspase-3/7 was biochemically confirmed; cells were cultured on 96-well plates (apo-ONE ${ }^{\circledR}$ Homogeneous Caspase-3/7 kit, Promega, Madison, WI, USA) following the manufacturer's instructions, and then the fluorescence intensity was measured using a fluorescence plate reader (FLx 800 Microplate, BIO-TEK Instruments, Winooski, VT, USA). The amount of fluorescent product generated is proportional to the amount of caspase-3/7 cleavage activity in the sample and is expressed as relative fluorescence units.

\section{Statistical analysis}

Statistical analysis was performed by a statistician Hisayosi Kondo (HK). Statistical differences in 53BP1 expression among different groups were compared using one-way analysis of variance (ANOVA) followed by post-hoc test (using the Bonferroni method) or two-way ANOVA followed by posthoc test (using the Bonferroni method). The Jonckheere test was used to assess the associations between 53BP1 expression type and pathological factors of nonalcoholic fatty liver disease. Spearman's rank correlation was employed to investigate the association between 53BP1 expression type and clinical features. All statistical tests were two-sided, and $p<0.05$ was considered statistically significant. The calculations were performed using the GLM, FREQ, CORR, and LOGISTIC procedures in the SAS software package (version 9.3; SAS Institute, Inc., Cary, NC, USA).

\section{Results}

\section{BP1 expression in nonalcoholic fatty liver disease patients}

Compared to that in normal tissue, the number of abnormal type of 53BP1-positive foci was significantly increased in nonalcoholic fatty liver hepatocytes $(p<0.01)$. However, abnormal foci expression did not show any difference between nonalcoholic fatty liver and nonalcoholic steatohepatitis (Fig. 2a, b). In contrast, the number of large 53BP1-positive foci was significantly higher in the nonalcoholic steatohepatitis patients than in the nonalcoholic fatty liver patients $(p<0.05)$, whereas no statistical difference was observed between normal and nonalcoholic fatty liver (Fig. 2b).

To ascertain that 53BP1 expression reflects DDR pathway activation in human nonalcoholic steatohepatitis, the human liver samples were costained with 53BP1 and $\gamma \mathrm{H} 2 \mathrm{AX}$, a known marker of DDR. In this analysis, 53BP1 partially colocalized with $\gamma \mathrm{H} 2 \mathrm{AX}$, further confirming the presence of DNA damage in nonalcoholic fatty liver disease patients (Fig. 2c). Notably, while the number of abnormal foci was not significantly affected by the presence or absence of fibrosis in the nonalcoholic fatty liver disease patients $(22.9 \%$ and $17.1 \%$, respectively), the number of large foci was significantly increased in nonalcoholic fatty liver disease patients with fibrosis $(6.08 \%)$ compared to that in patients without fibrosis $(1.51 \%$; $p<$ 0.01) (Fig. 2d). Based on our results, association of 53BP1-positive large foci with nonalcoholic fatty liver disease patients who would or would not have nonalcoholic steatohepatitis (by Matteoni classification) was reflected by an area under the curve of 0.752 (95\% confidence interval: 0.593-0.911). In addition, association of 53BP1-positive large foci with fibrosis was reflected by an area under the curve of 0.814 (95\% confidence interval: 0.672-0.957). By application of ROC information, cutoff values for further screening can be set based on the number of 53BP1-positive large foci. For example, identifying nonalcoholic steatohepatitis/fibrosis cases at risk using a cutoff value for the proportion of 53BP1-positive large foci, $2.47 \% / 2.69 \%$, would yield a true-positive rate (sensitivity) of $0.649 / 0.727$.

We next examined the localization of 53BP1, as cellular damage, steatosis, and fibrosis predominantly occur in the peri-venous area (zone 3 ) in adult nonalcoholic fatty liver disease patients [30]. Somewhat surprisingly, 53Bp1 nuclear expression was similar between zones 1,2 , and 3 (Fig. 2e, Supplementary Figure 1). These results indicate that while 53BP1 expression represents DDR pathway activation, its expression is not limited to hepatocytes that are histologically damaged. 
Table 1 Clinical characteristics and histological classification of the nonalcoholic fatty liver disease patients enrolled in this study

\begin{tabular}{|c|c|c|c|c|}
\hline \multicolumn{3}{|l|}{ Clinical data } & \multicolumn{2}{|l|}{ Histology } \\
\hline Age & $51.9(28-69)$ & years & Steatosis & $2.00(13)$ \\
\hline Body mass index & $27.4(21.8-35.08)$ & & Lobular inflammation & $1.12(0-2)$ \\
\hline $\begin{array}{l}\text { Aspartate } \\
\text { aminotransferase }\end{array}$ & $71.6(13-274)$ & $\mathrm{U} / \mathrm{L}$ & Hepatocyte ballooning & $1.29(0-2)$ \\
\hline Alanine aminotransferase & $95.8(11-421)$ & $\mathrm{U} / \mathrm{L}$ & $\begin{array}{l}\text { Total nonalcoholic fatty liver disease } \\
\text { activity score }\end{array}$ & $4.40(1-8)$ \\
\hline C-reactive protein & $0.24(0.011-1.15)$ & $\mathrm{mg} / \mathrm{dL}$ & Fibrosis & $1.74(0-4)$ \\
\hline Ferritin & $321.0(7-1559)$ & $\mathrm{ng} / \mathrm{mL}$ & Portal inflammation & $0.93(0-2)$ \\
\hline Platelet count & $20.9(10.8-34.4)$ & $10^{4} / \mu \mathrm{m}$ & & \\
\hline
\end{tabular}

Nonalcoholic fatty liver disease activity score (range from 0 to 8 ) represents the sum of the degree of steatosis (0-3), lobular inflammation (0-3), and hepatocyte ballooning (0-2). Fibrosis scoring was performed according to the Nonalcoholic Steatohepatitis Clinical Research Network System as follows: 0 (absent), 1 (perisinusoidal/pericellular fibrosis), 2 (periportal fibrosis), 3 (bridging fibrosis), and 4 (cirrhosis). The degree of portal inflammation was assessed as described by Brunt ( 0 none; 1 mild; 2 moderate; or 3 severe). Data are given as the mean (range)

\section{Association between 53BP1 expression and nonalcoholic fatty liver disease clinicopathological factors}

Table 1 shows the clinical and pathological characteristics of the nonalcoholic fatty liver disease patients enrolled in this study. In our analysis of the association between 53BP1 expression and these clinicopathological factors, we observed that the formation of abnormal 53BP1-positive foci was significantly correlated with the level of portal inflammation $(p<0.05)$ (Table 2), which has been previously indicated as a marker of advanced nonalcoholic fatty liver disease [29]. However, hepatocyte ballooning, lobular inflammation, and steatosis were not related to abnormal foci formation. Notably, the large foci type was also positively associated with the level of portal inflammation $(p<0.05)$ as well as fibrosis score $(p<0.05)$ (Table 2, Fig. 3a). Steatosis also appears to have a somewhat positive relationship with the presence of large foci.

With regard to the serum measurements, both the abnormal type and large type of 53BP1-positive foci were significantly associated with increased age and lower platelet count, which are known clinical markers of fibrosis (Table 3, Fig. 3b) $[4,31]$. In addition, the formation of both abnormal and large foci was also related to serum C-reactive protein levels, an indicator of inflammation that is correlated with the severity of hepatic fibrosis in nonalcoholic fatty liver disease [32]. In contrast, the presence of 53PB1-positive foci was not associated with body mass index or ferritin levels.

\section{Caspase-dependent 53BP1 expression in cultured rat hepatocytes following palmitate treatment}

To determine the potential role of free fatty acids in inducing DNA damage, we treated cultured hepatocytes with palmitate, which plays a role in hepatocyte lipotoxicity and is the most abundant circulating free fatty acid in human. Palmitate has been shown to induce cell death in cultured hepatocytes at concentrations of $200-800 \mu \mathrm{M}$ in previous studies $[33,34]$. As our goal was to create an environment that activated the DDR without inducing cell death, we first confirmed that $100 \mu \mathrm{M}$ palmitate did not significantly induce toxicity in clone 9 cells after $24 \mathrm{~h}$ (Supplementary Figure 2). After confirming that this concentration does not induce cell death, we evaluated the number of 53BP1positive nuclear foci after a $24 \mathrm{~h}$ treatment. In clone 9 cells treated with vehicle, 53BP1 expression was observed in a few cell foci (Fig. 4a, b). However, when treated with $100 \mu \mathrm{M}$ palmitate, the number of abnormal 53BP1-positive foci was significantly increased (Fig. 4a, b), indicating that palmitate stimulation induces DDR in cultured rat primary hepatocytes. We further investigated the possible mechanism by which DDR was induced by palmitate. The previous reports indicated the crucial role of sublethal caspase-3 activation in inducing genomic instability in radiationexposed cells [35]. Interestingly, low-dose palmitate $(100 \mu \mathrm{M})$ did induce a modest increase of caspase-3/7 activation in rat primary clone 9 cells (Fig. 5a), while not inducing significant cell death. Furthermore, pan-caspase inhibitor zVad-fmk significantly reduced palmitate-induced abnormal foci of 53BP1 (Fig. 5b). Thus, palmitate mediated53BP1 nuclear foci formation is caspase dependent.

\section{Discussion}

In the present study, we used 53BP1 immunofluorescence to evaluate DDR during nonalcoholic fatty liver disease and in free fatty acid-treated clone 9 cells. Our results indicate abnormal 53BP1 expression in nonalcoholic fatty liver disease tissues from an early stage, and that the occurrence of large 53BP1-positive foci is associated with the degree of 
Table 2 Association between nuclear 53BP1 expression and pathological classification in nonalcoholic fatty liver disease patients

\begin{tabular}{|c|c|c|c|}
\hline Fibrosis & $n$ & $\begin{array}{l}\text { Abnormal 53BP1 } \\
\text { foci }(\%)\end{array}$ & $\begin{array}{l}\text { Large 53BP1 } \\
\text { foci }(\%)\end{array}$ \\
\hline 0 & 8 & $17.1 \pm 10.2$ & $1.5 \pm 1.6$ \\
\hline 1 & 15 & $22.8 \pm 18.9$ & $5.2 \pm 4.1$ \\
\hline 2 & 4 & $18.0 \pm 10.7$ & $7.5 \pm 6.5$ \\
\hline 3 & 10 & $23.3 \pm 15.3$ & $6.2 \pm 3.9$ \\
\hline 4 & 5 & $\begin{array}{l}26.4 \pm 16.2 \\
\text { n.s. }\end{array}$ & $\begin{array}{l}7.6 \pm 3.2 \\
p<0.01\end{array}$ \\
\hline Portal inflammation & $n$ & $\begin{array}{l}\text { Abnormal 53BP1 } \\
\text { foci }(\%)\end{array}$ & $\begin{array}{l}\text { Large } \\
\text { 53BP1 foci } \\
(\%)\end{array}$ \\
\hline 0 & 10 & $12.6 \pm 8.6$ & $2.5 \pm 2.9$ \\
\hline 1 & 26 & $22.0 \pm 16.5$ & $6.1 \pm 4.7$ \\
\hline 2 & 6 & $\begin{array}{l}37.8 \pm 9.5 \\
p<0.05\end{array}$ & $\begin{array}{l}6.4 \pm 2.8 \\
p<0.05\end{array}$ \\
\hline Steatosis & $n$ & $\begin{array}{l}\text { Abnormal 53BP1 } \\
\text { foci }(\%)\end{array}$ & $\begin{array}{l}\text { Large } \\
\text { 53BP1 foci } \\
(\%)\end{array}$ \\
\hline 1 & 14 & $22.2 \pm 12.9$ & $3.4 \pm 3.7$ \\
\hline 2 & 14 & $26.5 \pm 18.4$ & $5.3 \pm 3.1$ \\
\hline 3 & 14 & $\begin{array}{l}16.7 \pm 14.4 \\
\text { n.s. }\end{array}$ & $\begin{array}{l}6.9 \pm 5.3 \\
p<0.05\end{array}$ \\
\hline Hepatocyte ballooning & $n$ & $\begin{array}{l}\text { Abnormal 53BP1 } \\
\text { foci }(\%)\end{array}$ & $\begin{array}{l}\text { Large } \\
53 B P 1 \text { foci } \\
(\%)\end{array}$ \\
\hline 0 & 5 & $17.5 \pm 13.5$ & $1.8 \pm 1.7$ \\
\hline 1 & 20 & $23.8 \pm 16.3$ & $6.4 \pm 5.3$ \\
\hline 2 & 17 & $\begin{array}{l}20.7 \pm 15.7 \\
\text { n.s. }\end{array}$ & $\begin{array}{l}4.8 \pm 2.9 \\
\text { n.s. }\end{array}$ \\
\hline Lobular inflammation & $n$ & $\begin{array}{l}\text { Abnormal 53BP1 } \\
\text { foci }(\%)\end{array}$ & $\begin{array}{l}\text { Large } \\
\text { 53BP1 foci } \\
(\%)\end{array}$ \\
\hline 0 & 5 & $9.3 \pm 5.6$ & $2.1 \pm 1.7$ \\
\hline 1 & 28 & $22.7 \pm 17.0$ & $5.8 \pm 4.6$ \\
\hline 2 & 9 & $\begin{array}{l}25.9 \pm 12.7 \\
\text { n.s. }\end{array}$ & $\begin{array}{l}5.0 \pm 4.0 \\
\text { n.s. }\end{array}$ \\
\hline $\begin{array}{l}\text { Nonalcoholic fatty liver } \\
\text { disease activity score total }\end{array}$ & $n$ & $\begin{array}{l}\text { Abnormal 53BP1 } \\
\text { foci }(\%)\end{array}$ & $\begin{array}{l}\text { Large } \\
\text { 53BP1 foci } \\
(\%)\end{array}$ \\
\hline 1 & 3 & $11.2 \pm 5.5$ & $1.3 \pm 0.92$ \\
\hline 2 & 1 & $24.4 \pm 0$ & $5.6 \pm 0$ \\
\hline 3 & 7 & $25.8 \pm 13.6$ & $4.9 \pm 4.3$ \\
\hline 4 & 10 & $25.3 \pm 15.3$ & $4.8 \pm 3.4$ \\
\hline 5 & 10 & $22.8 \pm 21.8$ & $7.4 \pm 5.5$ \\
\hline 6 & 6 & $17.6 \pm 10.5$ & $5.8 \pm 3.7$ \\
\hline 7 & 4 & $19.2 \pm 12.3$ & $5.5 \pm 3.8$ \\
\hline 8 & 1 & $\begin{array}{l}21.8 \pm 0 \\
\text { n.s. }\end{array}$ & $\begin{array}{l}2.7 \pm 0 \\
\text { n.s. }\end{array}$ \\
\hline
\end{tabular}

fibrosis. In cell culture, treatment with palmitate, the most highly expressed free fatty acid in humans, induced the formation of 53BP1-positive nuclear foci in clone 9 hepatocytes, indicating that free fatty acids may play a role in the mechanism underlying 53BP1 nuclear foci formation during nonalcoholic fatty liver disease pathogenesis.

DDR molecules have been investigated in the development of nonalcoholic fatty liver disease in various animal models. For example, a methionine- and choline-deficient diet-induced nonalcoholic fatty liver disease mouse model was found to have increased expression of Gadd45a, a crucial protein in the DDR pathway and genome stability. Gadd45 deficiency triggers worsening of nonalcoholic steatohepatitis in mice, indicating that DDR defects and subsequent genomic instability are involved in disease progression [36]. In addition, a recent study showed hepatocyte polyploidation, defined as cells containing more than two paired homologous sets of chromosomes [37], in both nonalcoholic fatty liver disease patients and a mouse model of the disease [38]. This phenomenon was aligned with the activity of other DDR proteins, such as the ataxia telangiectasia-mutated protein, in mouse hepatocytes. More importantly, abnormal hepatocyte polyploidation was increased in the livers of nonalcoholic fatty liver disease patients with hepatocellular carcinoma compared to noncarcinoma bearing human livers, indicating a strong relationship between genomic instability, polyploidation, and carcinogenesis in nonalcoholic fatty liver disease. Our current study is in concert with these previous reports and relationships between these characteristics as well as 53BP1 expression.

In the present study, an increased number of abnormal 53BP1-positive foci less than $1 \mu \mathrm{m}$ in size was observed in early nonalcoholic fatty liver disease without fibrosis as well as in advanced nonalcoholic fatty liver disease with fibrosis. These results suggest the occurrence of substantial genomic instability in the hepatocytes of nonalcoholic fatty liver disease cases even in the absence of advanced fibrosis. Pathologic changes associated with nonalcoholic fatty liver disease, such as the ballooning of hepatocytes, generally begin at the peri-central vein in adult nonalcoholic fatty liver disease patients [39, 40]. However, in our study, 53BP1-positive nuclear foci were distributed not only in the peri-central vein but also in both the intermediate and periportal zones, indicating that double-strand breaks occur independent of the histologically visible damage.

Because the formation of large 53BP1-positive foci is thought to be triggered by the marked accumulation of 53BP1 molecules [15, 17], formation of these foci may indicate a failure or prolongation of efficient recovery after a double-strand break. One of the key findings of our study is that large foci formation is significantly associated with pathological fibrosis in the liver of nonalcoholic fatty liver disease patients. The degree of portal inflammation, which is thought to precede fibrosis, was also found to be 
Fig. 3 Association of 53BP1positive nuclear foci with liver fibrosis and platelet count. a Positive association of hepatocyte p53-binding protein 1 (53BP1)-positive nuclear large foci expression with fibrosis stage in nonalcoholic fatty liver disease patients. The median values are indicated by the horizontal bars in the scatter plot. In the box plot, the bottom and top of the box are the 25th and 75th percentiles (the lower and upper quartiles), respectively. The top and bottom of the bar represents the highest and lowest data points, respectively. $p<0.05$

(Jonckheere test). b Negative association of hepatocyte 53BP1-positive abnormal foci (upper panel, $p<0.05$, Spearman's rank correlation) and large foci expression (lower panel, $p<0.05$, Spearman's rank correlation) with platelet count in nonalcoholic fatty liver disease patients (a)

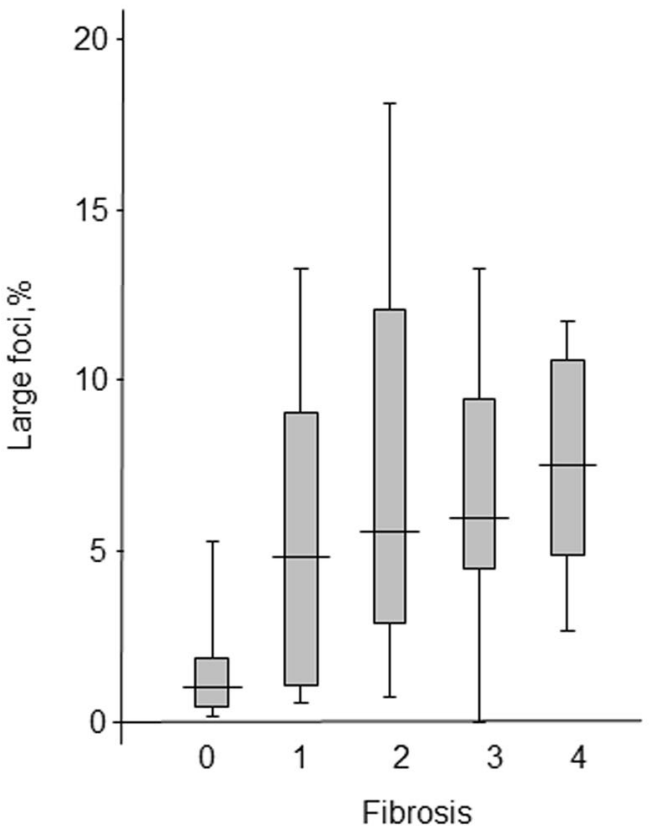

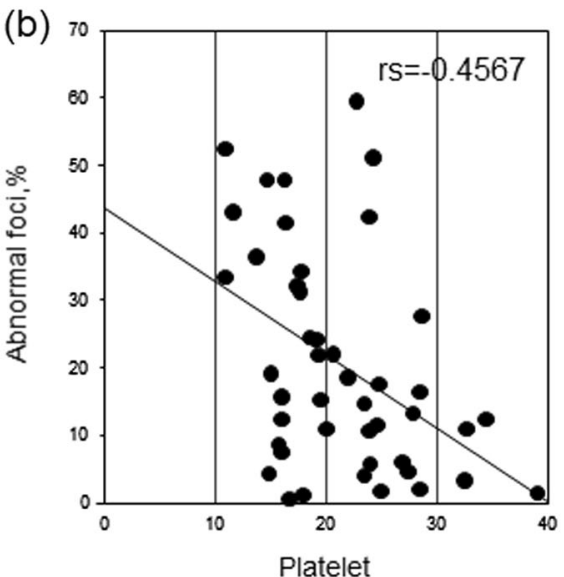

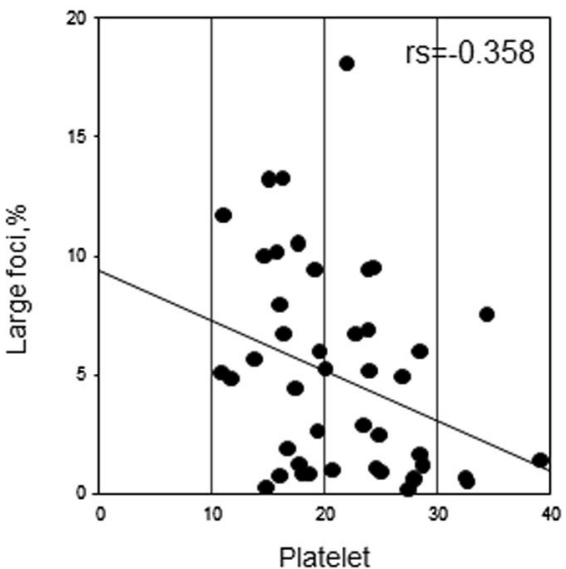

Table 3 Association between nuclear 53BP1 expression and clinical characteristics in nonalcoholic fatty liver disease patients

\begin{tabular}{lll}
\hline & \multicolumn{2}{l}{ Correlation coefficient } \\
\cline { 2 - 3 } & $\begin{array}{l}\text { Abnormal 53BP1 } \\
\text { foci }\end{array}$ & $\begin{array}{l}\text { Large 53BP1 } \\
\text { foci }\end{array}$ \\
\hline Age & $0.305^{*}$ & $0.4603^{* *}$ \\
Body mass index & 0.1497 & 0.1531 \\
Aspartate aminotransferase & $0.0103^{*}$ & $0.0060^{*}$ \\
Alanine aminotransferase & -0.921 & 0.0696 \\
C-reactive protein & $0.2013^{*}$ & $0.1849^{*}$ \\
Ferritin & 0.0211 & 0.1447 \\
Platelet count & $-0.4567^{* *}$ & $-0.358^{*}$
\end{tabular}

The correlation coefficient determined by Spearman's test is shown. $* p<0.05 . * * p<0.01$

associated with large 53BP1-positive foci. These findings were supported by the correlation between both abnormal and large foci types and platelet count, which decreases upon loss of functioning hepatocytes during progression of fibrosis.

Further, we also examined the expression of 53BP1 in five nonalcoholic fatty liver disease patients who later developed hepatocellular carcinoma during follow-up (Supplementary Table 1, Supplementary Fig. 3). Interestingly, two out of the six patients had relatively high numbers of large 53BP1-positive foci-case 5: $18 \%$, case 6: $12 \%$-before developing hepatocellular carcinoma. The 53BP1 expression in case 5 was the highest among all the cases in the current study. We also noted that case 6 had relatively high 53BP1 expression despite the low fibrosis score (F1) (Supplementary Table 1). In contrast, the other four cases (cases 1-4) had relatively low expression of 53BP1, which indicates that 53BP1 expression cannot solely explain the risk of hepatocellular carcinoma. Nevertheless, we consider that close monitoring may be required for cases that present high numbers of large 53BP1-positive foci, regardless of the fibrosis state.

This study on nonalcoholic fatty liver disease patients presents a preliminary assessment of a generic form of DNA damage; other ongoing studies will evaluate other forms of chronic liver disease. Our preliminary study indicates increased 53BP1 expression, in both abnormal and large foci, in chronic hepatitis C (Supplementary Figure 4). We also noted that five cases of acute liver dysfunction presented with increased numbers of abnormal 53BP1-positive foci compared to the normal control, but did not present 
Fig. 4 p53-binding protein 1 (53BP1) expression is induced by free fatty acid treatment. a Expression of 53BP1 in clone 9 rat hepatocytes after treatment with $100 \mu \mathrm{M}$ palmitate for $24 \mathrm{~h}$. Untreated cells were used as a control. b Quantification of the abnormal 53BP1 foci with and without palmitate treatment. Data are expressed as the means \pm SEM. $* p<0.05$
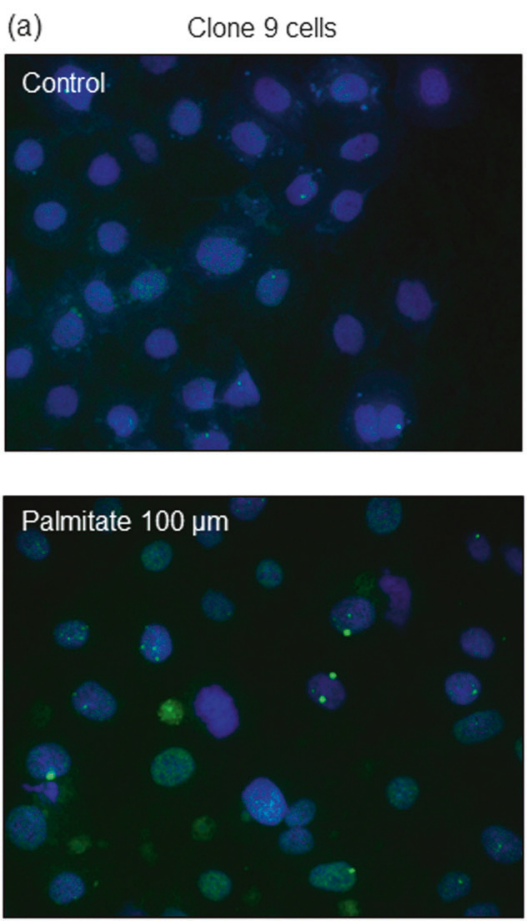

(b)

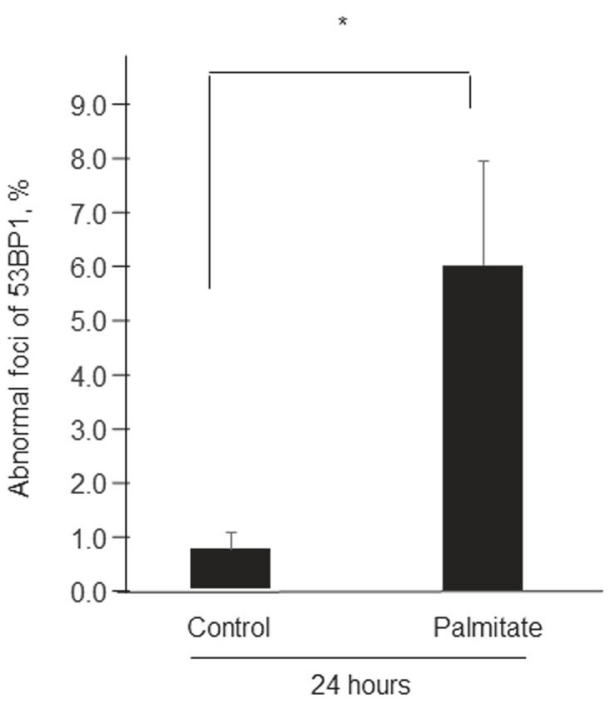

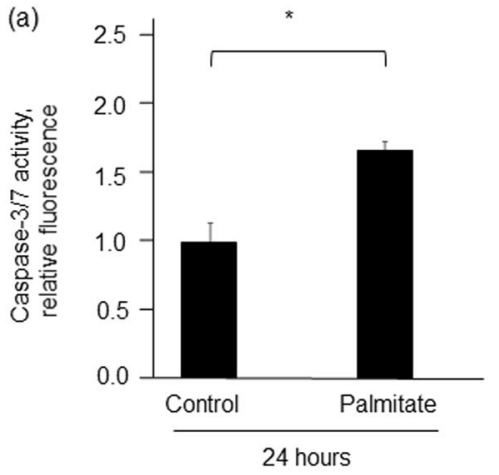

Fig. 5 Caspase-independent expression of p53-binding protein 1 (53BP1) in palmitate-treated hepatocytes. Data are expressed as the means \pm SEM. $* p<0.05$. a Caspase activity assay in clone 9 rat hepatocytes after treatment with $100 \mu \mathrm{M}$ palmitate for $24 \mathrm{~h}$. b Cells

large foci (Supplementary Table 2). Positive correlation was found between large foci and age in nonalcoholic fatty liver disease patients. Age is another strong risk factor for DNA instability and carcinogenesis. Although the average age was higher in the normal group compared to the nonalcoholic fatty liver disease group, minimal 53BP1 expression was observed in the healthy liver. Thus, it is unlikely that 53BP1 expression is solely determined by age, but rather occurs in combination with nonalcoholic fatty liver disease pathogenesis and aging.

It is widely known that increased free fatty acid levels and hepatocyte apoptosis are involved in nonalcoholic fatty liver disease pathogenesis [41]. Additional factors, such as

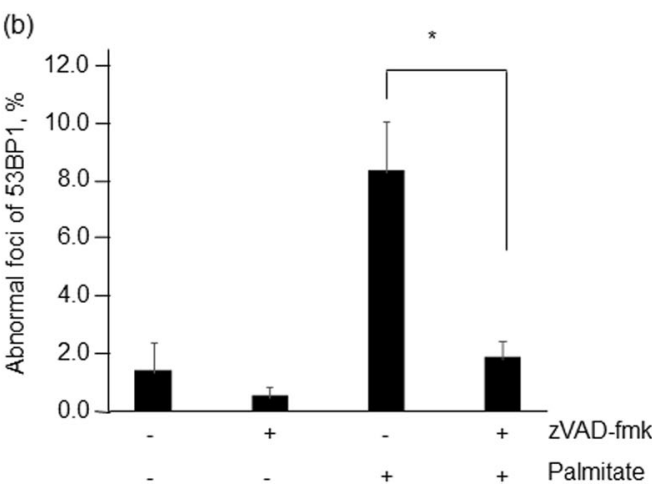

were treated with $100 \mu \mathrm{M}$ palmitate in presence or absence of caspase inhibitor $\mathrm{zVad}$-fmk $(10 \mu \mathrm{g} / \mathrm{mL})$. Rate of abnormal 53BP1 foci -expressing cells was quantified. Data are expressed as the means \pm SEM. $* p<0.05$

lipopolysaccharide, cholesterol, and other lipid derivatives, may also be significant causative factors of hepatocyte injury during nonalcoholic fatty liver disease [19, 24, 42, 43]. Our results indicate that palmitate treatment increases the formation of abnormal 53BP1-positive foci. Although apoptosis is a key feature of nonalcoholic fatty liver disease and could be related to the free fatty acid content [41], the palmitate concentration used in our treatments was not sufficient to induce apoptosis. This allowed us to focus on the effect of palmitate on double-strand break-related 53BP1 expression. Our results indicate that at the sublethal palmitate concentration, cells are able to survive, but the caspase-dependent DDR pathway is activated. This 
observation is similar to that of Liu et al. [35], which also demonstrated that cells exposed to a minimal dose of ionizing radiation can survive despite caspase- 3 activation, and then possibly facilitate carcinogenesis by inducing genetic instability. Additional studies in a large population are required to identify how the free fatty acid-induced DDR leads to DNA instability and should include analysis of chromosomal re-arrangement by fluorescence in situ hybridization (FISH), DNA copy number aberration by microarray, and DNA methylation and microRNAs by nextgeneration sequencing. Upon further analysis in a larger sample size, our findings can be feasibly applied for estimating the risk of cancer development in nonalcoholic fatty liver disease patients.

In conclusion, we analyzed 53BP1 nuclear foci formation in relation to DDR in nonalcoholic fatty liver disease patients in the early phase of the disease as well as the mechanism underlying foci formation. Our data indicate that not only does the formation of 53BP1-positive foci increase during nonalcoholic fatty liver disease, but also the number of large foci is correlated with the level of fibrosis. Furthermore, our results also show that the formation of these 53BP1-expressing foci is induced, at least in part, by saturated free fatty acid-induced caspase activation. Taken together, the results of this study demonstrate a role for 53BP1 in nonalcoholic fatty liver disease in vivo in addition to highlighting the role of free fatty acids in the induction of double-strand breaks and 53BP1 expression in vitro. While additional work is necessary, we believe that detection of 53BP1-positive foci could be used as an additional tool for estimating the magnitude of DDR activation in nonalcoholic fatty liver disease patients.

Acknowledgements This work was supported by a grant from JSPS KAKENHI (grant number 24790709) and by Program of the NetworkType Joint Usage/Research Center for Radiation Disaster Medical Science. We thank Dr. Shuji Toda, Department of Pathology, Faculty of Medicine, Saga University, for providing the samples, and Dr. Ryu Sasaki, Department of Gastroenterology and Hepatology, Nagasaki University Hospital, for figure arrangement. We thank Yoko Kido, Yasuko Kanda, and Mami Morishita, and Shu Nagatomo for their technical support.

\section{Compliance with ethical standards}

Conflict of interest The authors declare that they have no conflict of interest.

Publisher's note: Springer Nature remains neutral with regard to jurisdictional claims in published maps and institutional affiliations.

\section{References}

1. Romero-Gómez M, Zelber-Sagi STrenell M Treatment of NAFLD with diet, physical activity and exercise. J Hepatol. 2017.
2. Adams LA, Lymp JF, St Sauver J, Sanderson SO, Lindor KD, Feldstein A, et al. The natural history of nonalcoholic fatty liver disease: a population-based cohort study. Gastroenterology. 2005;129:113-21.

3. Sorrentino P, Tarantino G, Perrella A, Micheli P, Perrella O, Conca $\mathrm{P}$, et al. A clinical-morphological study on cholestatic presentation of nonalcoholic fatty liver disease. Dig Dis Sci. 2005;50:1130-5.

4. Hagstrom H, Nasr P, Ekstedt M, Hammar U, Stal P, Hultcrantz R, et al. Fibrosis stage but not NASH predicts mortality and time to development of severe liver disease in biopsy-proven NAFLD. J Hepatol. 2017;67:1265-73.

5. Baffy G, Brunt EMCaldwell SH. Hepatocellular carcinoma in non-alcoholic fatty liver disease: an emerging menace. J Hepatol. 2012;56:1384-91.

6. Ferguson LR, Chen H, Collins AR, Connell M, Damia G, Dasgupta S, et al. Genomic instability in human cancer: Molecular insights and opportunities for therapeutic attack and prevention through diet and nutrition. Semin Cancer Biol. 2015;35:Suppl: S5-24.

7. Aparicio T, Baer RGautier J. DNA double-strand break repair pathway choice and cancer. DNA Repair (Amst). 2014;19:169-175.

8. Barash H, R Gross E, Edrei Y, Ella E, Israel A, Cohen I, et al. Accelerated carcinogenesis following liver regeneration is associated with chronic inflammation-induced double-strand DNA breaks. Proc Natl Acad Sci U S A. 2010;107:2207-12.

9. Langevin F, Crossan GP, Rosado IV, Arends MJ, Patel KJ. Fancd 2 counteracts the toxic effects of naturally produced aldehydes in mice. Nature. 2011;475:53-8.

10. Minakawa Y, Atsumi Y, Shinohara A, Murakami YYoshioka K. Gamma-irradiated quiescent cells repair directly induced doublestrand breaks but accumulate persistent double-strand breaks during subsequent DNA replication. Genes Cells. 2016;21:789-97.

11. Bork P, Hofmann K, Bucher P, Neuwald AF, Altschul SFKoonin EV. A superfamily of conserved domains in DNA damageresponsive cell cycle checkpoint proteins. FASEB J. 1997;11:68-76.

12. Drane P, Brault ME, Cui G, Meghani K, Chaubey S, Detappe A, et al. TIRR regulates 53BP1 by masking its histone methyl-lysine binding function. Nature. 2017;543:211-6.

13. Zhang A, Peng B, Huang P, Chen J, Gong Z. The p53-binding protein 1-Tudor-interacting repair regulator complex participates in the DNA damage response. J Biol Chem. 2017;292:6461-6467.

14. Thorslund T, Ripplinger A, Hoffmann S, Wild T, Uckelmann M, Villumsen B, et al. Histone $\mathrm{H} 1$ couples initiation and amplification of ubiquitin signalling after DNA damage. Nature. 2015;527:389-93.

15. Nakashima M, Suzuki K, Meirmanov S, Naruke Y, MatsuuMatsuyama M, Shichijo K, et al. Foci formation of P53-binding protein 1 in thyroid tumors: activation of genomic instability during thyroid carcinogenesis. Int J Cancer. 2008;122:1082-1088.

16. Lanton T, Shriki A, Nechemia-Arbely Y, Abramovitch R, Levkovitch O, Adar R, et al. Interleukin 6-dependent genomic instability heralds accelerated carcinogenesis following liver regeneration on a background of chronic hepatitis. Hepatology. 2017;65:1600-11.

17. Matsuda K, Miura S, Kurashige T, Suzuki K, Kondo H, Ihara M, et al. Significance of p53-binding protein 1 nuclear foci in uterine cervical lesions: endogenous DNA double strand breaks and genomic instability during carcinogenesis. Histopathology. 2011;59:441-51.

18. Mussazhanova Z, Akazawa Y, Matsuda K, Shichijo K, Miura S, Otsubo R, et al. Association between p53-binding protein 1 expression and genomic instability in oncocytic follicular adenoma of the thyroid. Endocr J. 2016;63:457-67. 
19. Akazawa Y Nakao K. To die or not to die: death signaling in nonalcoholic fatty liver disease. J Gastroenterol. 2018.

20. Feldstein AE, Wieckowska A, Lopez AR, Liu YC, Zein NN, et al. Cytokeratin-18 fragment levels as noninvasive biomarkers for nonalcoholic steatohepatitis: a multicenter validation study. Hepatology. 2009;50:1072-8.

21. Akazawa Y, Cazanave S, Mott JL, Elmi N, Bronk SF, Kohno S, et al. Palmitoleate attenuates palmitate-induced Bim and PUMA up-regulation and hepatocyte lipoapoptosis. J Hepatol. 2010;52:586-93.

22. Hirsova P, Ibrahim SH, Gores GJMalhi H. Lipotoxic lethal and sublethal stress signaling in hepatocytes: relevance to NASH pathogenesis. J Lipid Res. 2016;57:1758-70.

23. Wree A, Kahraman A, Gerken GCanbay A. Obesity affects the liver - the link between adipocytes and hepatocytes. Digestion. 2011;83:124-33.

24. Musso G, Cassader M, Paschetta E, Gambino R. Bioactive Lipid Species and Metabolic Pathways in Progression and Resolution of Nonalcoholic Steatohepatitis. Gastroenterology. 2018;155: 282-302.e288.

25. Cazanave SC, Mott JL, Elmi NA, Bronk SF, Werneburg NW, Akazawa Y, et al. JNK1-dependent PUMA expression contributes to hepatocyte lipoapoptosis. J Biol Chem. 2009;284:26591-602.

26. Akazawa Y, Nakao K. Lipotoxicity pathways intersect in hepatocytes: Endoplasmic reticulum stress, c-Jun N-terminal kinase-1, and death receptors. Hepatol Res. 2016;46:977-84.

27. Matteoni CA, Younossi ZM, Gramlich T, Boparai N, Liu YCMccullough AJ. Nonalcoholic fatty liver disease: a spectrum of clinical and pathological severity. Gastroenterology. 1999;116:1413-9.

28. Kleiner DE, Brunt EM, Van Natta M, Behling C, Contos MJ, Cummings OW, et al. Design and validation of a histological scoring system for nonalcoholic fatty liver disease. Hepatology. 2005;41:1313-21.

29. Brunt EM, Kleiner DE, Wilson LA, Unalp A, Behling CE, Lavine JE, et al. Portal chronic inflammation in nonalcoholic fatty liver disease (NAFLD): a histologic marker of advanced NAFLD-Clinicopathologic correlations from the nonalcoholic steatohepatitis clinical research network. Hepatology. 2009;49: 809-20.

30. Brunt EM, Ramrakhiani S, Cordes BG, Neuschwander-Tetri BA, Janney CG, Bacon BR, et al. Concurrence of histologic features of steatohepatitis with other forms of chronic liver disease. Mod Pathol. 2003;16:49-56.

31. Kawamura Y, Arase Y, Ikeda K, Seko Y, Imai N, Hosaka T, et al. Large-scale long-term follow-up study of Japanese patients with non-alcoholic Fatty liver disease for the onset of hepatocellular carcinoma. Am J Gastroenterol. 2012;107:253-61.
32. Yoneda M, Mawatari H, Fujita K, Iida H, Yonemitsu K, Kato S, et al. High-sensitivity C-reactive protein is an independent clinical feature of nonalcoholic steatohepatitis (NASH) and also of the severity of fibrosis in NASH. J Gastroenterol. 2007; 42:573-82.

33. Akazawa Y, Guicciardi ME, Cazanave SC, Bronk SF, Werneburg NW, Kakisaka K, et al. Degradation of cIAPs contributes to hepatocyte lipoapoptosis. Am J Physiol Gastrointest Liver Physiol. 2013;305:G611-9.

34. Cazanave SC, Wang X, Zhou H, Rahmani M, Grant S, Durrant DE, et al. Degradation of Keap1 activates BH3-only proteins Bim and PUMA during hepatocyte lipoapoptosis. Cell Death Differ. 2014;21:1303-12.

35. Liu X, He Y, Li F, Huang Q, Kato TA, Hall RP, et al. Caspase-3 promotes genetic instability and carcinogenesis. Molecular cell. 2015;58:284-96.

36. Tanaka N, Takahashi S, Hu X, Yu L, Fujimori N, Golla S, et al. Growth arrest and DNA damage-inducible $45 \alpha$ protects against nonalcoholic steatohepatitis induced by methionineand choline-deficient diet. Biochim Biophys Acta. 2017; 1863:3170-82. https://doi.org/10.1016/j.bbadis.2017.08.017. Epub 2017 Aug 24.

37. Gentric GDesdouets C. Polyploidization in liver tissue. Am J Pathol. 2014;184:322-31.

38. Gentric G, Maillet V, Paradis V, Couton D, L'hermitte A, Panasyuk $\mathrm{G}$, et al. Oxidative stress promotes pathologic polyploidization in nonalcoholic fatty liver disease. J Clin Invest. 2015;125:981-92.

39. Brunt EM. Pathology of fatty liver disease. Mod Pathol. 2007;20 (Suppl 1):S40-8.

40. Chalasani N, Younossi Z, Lavine JE, Diehl AM, Brunt EM, Cusi $\mathrm{K}$, et al. The diagnosis and management of non-alcoholic fatty liver disease: practice Guideline by the American Association for the Study of Liver Diseases, American College of Gastroenterology, and the American Gastroenterological Association. Hepatology. 2012;55:2005-23.

41. Feldstein AE, Canbay A, Angulo P, Taniai M, Burgart LJ, Lindor $\mathrm{KD}$, et al. Hepatocyte apoptosis and fas expression are prominent features of human nonalcoholic steatohepatitis. Gastroenterology. 2003; 125:437-43

42. Kudo H, Takahara T, Yata Y, Kawai K, Zhang W, Sugiyama T. Lipopolysaccharide triggered TNF-alpha-induced hepatocyte apoptosis in a murine non-alcoholic steatohepatitis model. J Hepatol. 2009;51:168-75.

43. Kakisaka K, Cazanave SC, Fingas CD, Guicciardi ME, Bronk SF, Werneburg NW, et al. Mechanisms of lysophosphatidylcholineinduced hepatocyte lipoapoptosis. Am J Physiol Gastrointest Liver Physiol. 2012;302:G77-84. 